\title{
Cost optimization estimation of medical institutions in grading diagnosis and treatment based on system dynamics model
}

\author{
Jiang Wu \\ Wuhan University \\ jiangwu@whu.edu.cn
}

\author{
Yao Yao \\ Wuhan University \\ 1204746416@qq.com
}

\author{
Xiao Huang* \\ Wuhan University \\ 851195240@qq.com
}

\begin{abstract}
The grading diagnosis and treatment system in China is to improve the first-time diagnosis rate of patients in primary health care institutions, thereby increasing the proportion of people in primary health care institutions, thus achieving the goal of reducing costs. This study constructs a system dynamics model of the number of primary and higher-level visits-the cost of medical institutions, and simulates the effect of the increase in the proportion of patients attending the primary level on the cost of medical institutions. The study found that with the increase in the number of visits, the cost of primary medical institutions will increase, but the total cost of the entire medical system will be reduced significantly. Moreover, the higher the proportion of the number of people attending the primary level, the lower the total cost. If the proportion of primary care in 2017 increases by 15\%, and this trend is maintained until 2021, the total cost saved by medical institutions in 2021 will be as high as 903.32 billion yuan.
\end{abstract}

\section{Introduction}

The grading diagnosis and treatment system is the focus of Chinese medical reform in recent years. The core of the system is to improve the service level of primary medical institutions, change the proportion of reimbursement for hospitals at different levels, and improve the proportion of patients who go to community hospitals, county hospitals and other primary hospitals. By means of the two-way referral method to implement the functions of acute and chronic disease diagnosis and treatment services of various types of medical institutions, so as to clarify the division of responsibilities of various types of medical and health institutions at all levels, then regulate the medical order. The specific problem addressed by the policy is that Chinese primary medical institutions have failed to meet enough medical needs, resulting in waste of medical resources in higher medical institutions.

The focus of grading diagnosis and treatment is to expand the service level of primary medical institutions. Specific measures include improving the quality of employees in primary medical institutions, and increasing the difference between the reimbursed proportion of primary medical institutions and higher medical institutions. These measures can attract more patients to the primary clinic of primary health care institutions, so that more patients with chronic diseases can be referred from the higher-level medical institutions to the primary medical institutions, thus increasing the number of visits to primary medical institutions. In short, this measure will enable primary medical institutions to serve a wider range of patients, and thus reduce the total cost of medical care in China. The reform measures can improve the number of visits to primary health care institutions. However, the details of the economic benefits of the increase in the number of visits to primary health care institutions are not known. This issue needs to be further verified. Scholars in many countries have established many models for medical-related research through system dynamics, with a focus on the evaluation of the effects of medical-related policies [1]. The impact of the increase in the number of visits to primary medical institutions on the cost of medical institutions can also be explored through system dynamics methods.

Therefore, this study will construct a system dynamics model for the number of visits to primary medical institutions, the number of visits to higher medical hospitals, and the cost of hospitals at different levels. Second, validate the validity of the model with real data. Finally, evaluate the impact of the increase in the number of visits to primary medical institutions in 2017-2021 on the total cost of Chinese medical institutions through sensitivity analysis.

\section{Literature review}


System dynamics methods are often used in policy evaluations, its medical-related applications are also related to policy. Such research mainly includes two types:(1) Assess the impact of macro policy changes on outcomes such as cost optimization. Ahmad S et al. assessed the cost-effectiveness of tobacco intervention control, finding that long-term savings in medical costs can offset the investment in tobacco intervention control, it also increases the life expectancy of the implementation of this policy area. $\mathrm{Yu}$ Wet al. predicted that adjustment of different health policies in China would affect the proportion of patients who did not seek medical care, the relationship between adjusting the number structure of hospitals and community hospitals, adjusting outpatient prices, and adjusting the level of health insurance to potential medical needs was found [2].Hungary introduced a new labor law in 2012, which allows informal payments to be accepted. Márta Somogyvári evaluated the impact of this policy on the country's health care industry and found that the policy created mistrust between doctors and patients and increased the total cost of social and health insurance funds [3].Ahmad, S assesses the policy that California hopes to implement -increasing the legal minimum purchase cigarette age to 21 - the cost-effectiveness of this policy, and found that after 50 years of implementation, the youth (14-17 years old) smoking rate $13.3 \%$ fell to $2.4 \%(82 \%$ reduction), actually saving the country and residents 24 billion US dollars [4]. (2) Assess the impact of different disease interventions. Edelstein B L et al. compared the potential outcomes of nine different preventive interventions for early childhood caries [5]. Because overuse of opioid analgesics is prone to death, Wakeland $\mathrm{W}$ et al. evaluated the impact of three different drug education interventions on the number of overuse deaths during the 2008-2015 evaluation period [6].

In addition, the system dynamics method is widely used in the assessment of medical resources supply and demand, mainly including the supply and demand of medical resources such as the supply and demand of doctors, the inventory of medical resources, and the simulation evaluation of the allocation of relevant resources in the crisis. Researchers from different countries have used the system dynamics method to simulate the number of doctors and supply and demand in the country. From Japan [7, 8], Spain [9], Canada [10] have found their country or a certain region within the country, they have found that the number of doctors will be in short supply at certain times. Wang L $\mathrm{C}$ et al. established a new hospital inventory demanddriven replenishment model that determines the optimal replenishment time and the total inventory cost [11]. The model developed by Senese F et al. predicts the evolution of the supply of Italian medical experts and combines demographics, service utilization and hospital beds to measure the potential effective allocation of medical allocations[12]. Diaz $\mathrm{R}$ et al. demonstrates the utility of the system dynamics approach to model and simulate US demand for ambulatory health care service both for the general population and for specific cohort subpopulations over the 5-year period, from 2003 to 2008. A system dynamics approach that is shown to meaningfully project demand for services has implications for health resource planning and for generating knowledge that is critical to assessing interventions[13]. Decisionmaking simulations in times of crisis are also the focus of researchers . Khanmohammadi $\mathrm{S}$ et al. proposed a system dynamics simulation model that describes the dynamic characteristics of the hospital's postearthquake recovery process, which enables managers to gain insight into how their decisions about available resource use before and after the earthquake affect hospital function, and Assist administrators in assessing the impact of various readiness policies on their hospital resilience [14]. Wenya $\mathrm{Y}$ et al. used the system dynamics method to establish a system dynamics model of mass casualties. The model, based on Shanghai, China, found that adjusting the efficiency of rescue ambulances and the allocation of emergency medical personnel to the efficiency of organization and command [15].

\section{Model building}

\subsection{The logic of model building}

At present, Chinese hospitals include primary medical institutions, second-tier medical institutions and third-tier medical institutions. The services of the primary medical institutions are significantly different from those of the second-tier and third-tier hospitals. For example, most of people choose inpatient services in higher-level hospitals, which leads to the tightness of beds in second-tier and third-tier hospitals, but not in primary hospitals. According to the relevant literature, news, and consulting experts in relevant fields, this study sorts out the relationship between the number of medical institutions and the cost of medical services at different levels, and completes the model construction with the system dynamics modeling tool Vensim, as shown in Figure 1. 


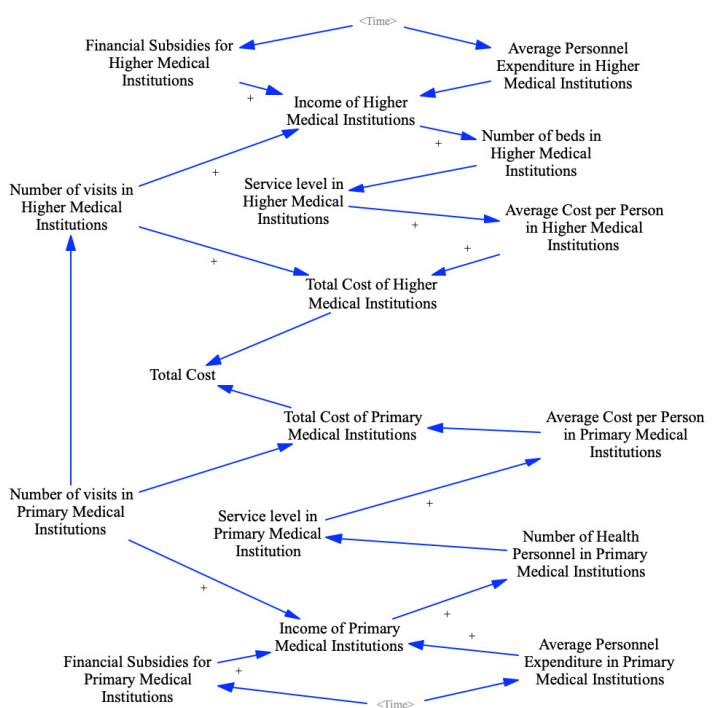

Figure.1. Logic model of the number of visits and medical expenses of medical institutions at different levels

The logical model of Figure 1 is drawn according to the actual situation, with the focus on improving referrals between patients in different levels of medical institutions. At the same time, the demographic model is introduced into the original model, as shown in Figure 2.

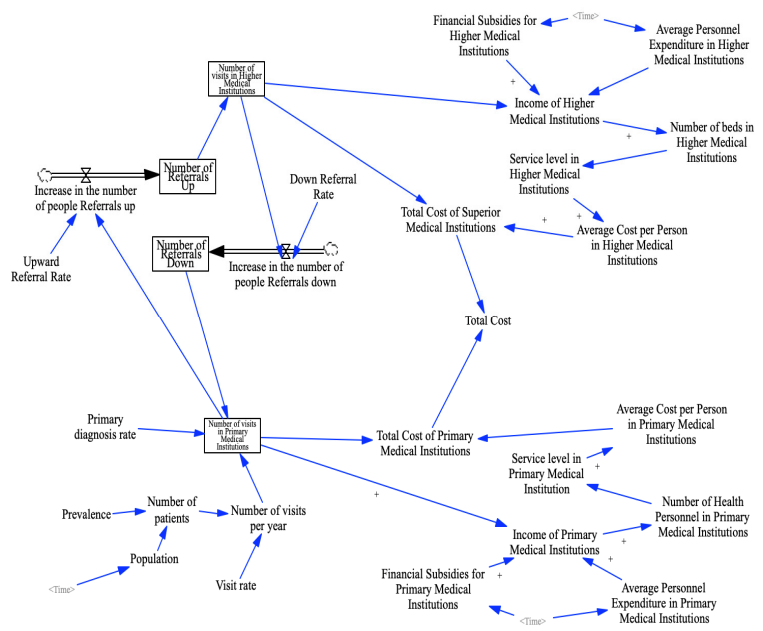

Figure.2. Actual model of the number of visits and medical expenses of medical institutions at different levels

As shown in Figure 2, the model consists of four subsystems, which are the primary medical institutions - the higher-level medical institution referral subsystem, the population-primary medical care visits number subsystem, primary medical institution's medical treatment-cost subsystem and the higher medical institution's medical treatment-cost subsystem. The four subsystems are separately divided to obtain four subsystem diagrams, as shown in Figure 3, Figure 4, and Figure 5.

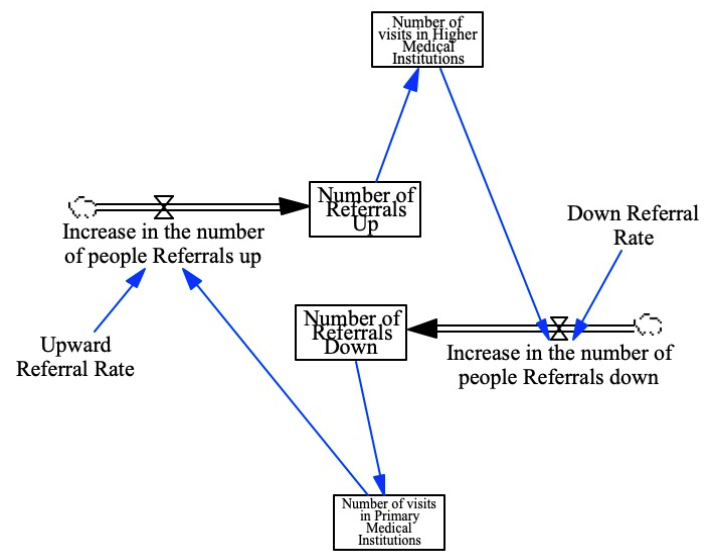

Figure.3. Subsidiary medical institution referral subsystem

Figure 3 shows the referral subsystem for the higher and primary medical institutions. The subsystem includes the stock and flow of the visit, and the patient at the primary care facility may refer to the patient, and the patient at the higher-level medical institution may refer to the patient.

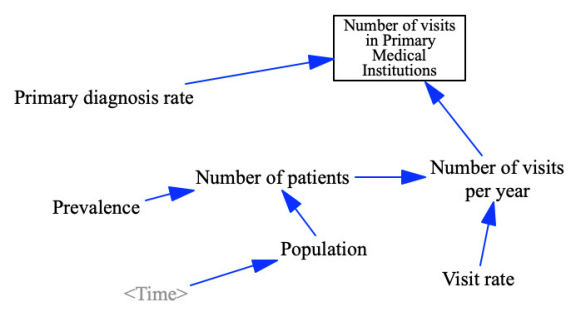

Figure.4. Population-basic patient visits subsystem

Figure 4 shows the population-basic patient visits subsystem. Changes in population over time will affect the number of people attending primary health care institutions, as well as the number of visits to higherlevel medical institutions. Changes in population will result in a change in the number of patients per year. In our model, the rate of visits to the primary level is certain, and the number of people attending primary care institutions will change. The difference between the total number of patients and the number of visits to 
primary care institutions is the number of visits to higher-level medical institutions.

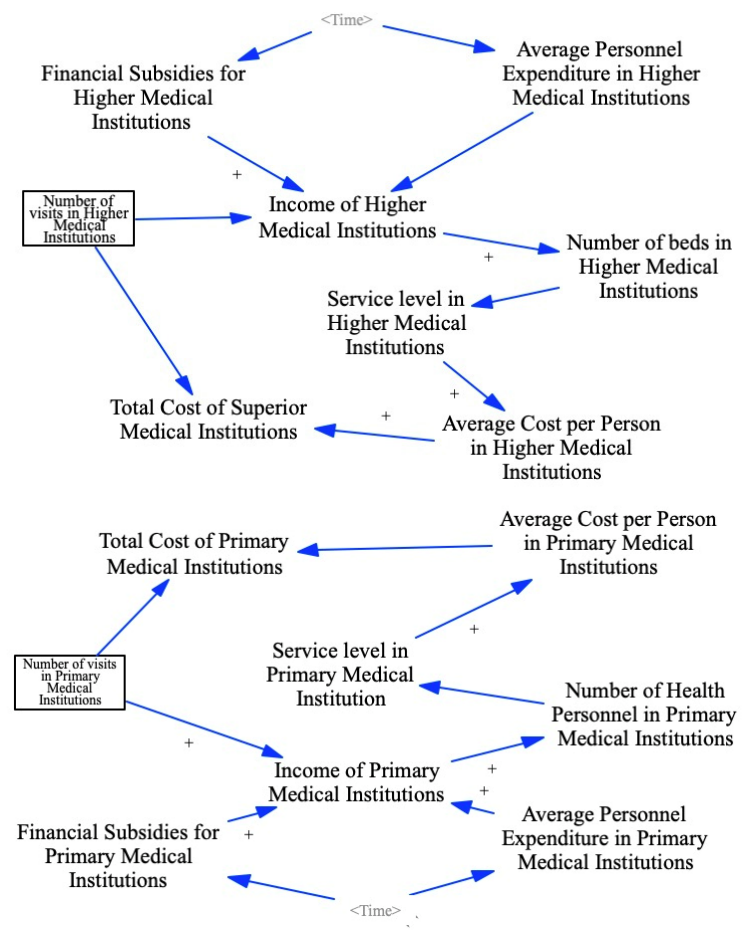

Figure.5. Higher and Primary medical base cost subsystem

As shown in Figure 5, our treatment-cost model consists of two subsystems, which are the primary medical institution's medical treatment-cost subsystem and the higher medical institution's medical treatmentcost subsystem. The number of visits to higher-level medical institutions will be reduced due to the diversion effect of primary medical institutions. In the subsystem of the higher medical institutions, the amount of medical treatment of the higher medical institutions and the per capita expenditure of the higher medical institutions jointly affect the income of the higher medical institutions. This part of the income together with the government financial subsidies constitutes the total income of the higher medical institutions. The accumulation of total annual income will have a positive impact on the number of beds in higher medical institutions in the next year. Thus, the service level of higher medical institutions will be improved, that is, the amount of medical care for the next year will be increased. The promotion has caused an increase in the per capita cost of the higher medical institutions. The per capita cost and the amount of medical treatment of the superior medical institutions jointly affect the total cost of the higher medical institutions. In the subsystems of primary medical institutions, there are similar relationships between variables, and ultimately caused changes in the total cost of primary medical institutions. In addition, since the per capita expenditure for medical treatment in primary medical institutions is lower than the per capita expenditure in higher medical institutions, as the flow of people from the higher medical institutions to the primary medical institutions, the total medical costs will be reduced, thereby increasing the utilization rate of medical resources.

\subsection{Model parameter relationship determination}

This study obtains various types of data of the above model from the 2014-2017 China Health Statistical Yearbook and the 2018 China Health and Wellness Statistical Yearbook, and combined with relevant demographic data and economic data, the parameter relationships in the above models are clarified. The parameters in the above model are divided into three categories:

(1) Variable relationship between the higher and primary referral subsystems and the population change subsystem. The relationship between the variables of the two subsystems does not change due to changes in the number of people attending the primary level.

Increase in the number of people Referrals down = Down Referral Rate*Number of visits in Higher Medical Institutions

Increase in the number of people Referrals up $=$ Number of visits in Primary Medical Institutions*Upward Referral Rate

Number of visits in Higher Medical Institutions = integ(Number of Referrals Up)+initial

Number of visits in Primary Medical Institutions = integ(Number of Referrals Down)+Number of visits per year* Primary diagnosis rate

Number of patients=Population*Prevalence

Number of visits per year=Number of patients*Visit rate

(2) The cost of primary medical institutions, the cost of higher medical institutions and the total cost. These parameters are directly calculated from the data in the model. Total Cost of Primary Medical Institutions $=$ Average Cost per Person in Primary Medical Institutions * Number of visits in Primary Medical Institutions; Total Cost of Higher Medical Institutions $=$ Average Cost per Person in Higher Medical Institutions * Number of visits in Higher Medical Institutions; Total cost $=$ Total Cost of Higher Medical Institutions + Total Cost of Primary Medical Institutions.

(3) Other various types of parameter relationships. In this study, the parameter relationship between the 
remaining parameters is clarified by least squares method, curve fitting and consulting the corresponding literature. For example, it is generally believed that the number of beds in a medical facility and the number of medical staffs will affect the number of visits to the medical institution. Therefore, the study initially determined that the service level of the medical institution was significantly correlated with the number of beds and the number of medical staffs, and the relationship between these variables was calculated by the least square method. However, it is found through calculation that the number of visits to higher hospitals is only significantly related to the number of beds, while the level of service of primary care institutions is only significantly related to the number of medical staffs. The most stressful resource in the higher hospital is the bed. Patients who are unwilling to see a doctor at the grassroots level often rely on the distrust of primary care workers and the lack of adequate medical staff at the primary health care facility to complete the consultation of most diseases. Therefore, the above results are consistent with the facts, so the factors related to the service level of different levels of hospitals are also different. It can be known that the service level of the superior medical institutions is significantly related to the number of beds, and the service level of the primary medical institutions is significantly related to the number of hospital personnel. Then, the relationship between these parameters is clarified by curve fitting with real data.

\subsection{Model test}

In this study, various system parameter relationships are uploaded to the above model to simulate the total cost of higher medical institutions and the total cost of primary medical institutions in the Chinese medical treatment system from 2013 to 2017. The simulation data is compared with the actual value to calculate the actual value of the higher-level medical institution and the cost of the primary medical institution and the error of the analog value. The results are shown in Table 1.

Table 1. Comparative analysis of medical institution cost simulation results and real results from 2013 to 2017

\begin{tabular}{|c|c|c|c|c|c|c|}
\hline \multirow[t]{2}{*}{ Year } & \multicolumn{3}{|c|}{$\begin{array}{c}\text { Higher level of medical } \\
\text { institutions (Billions of } \\
\text { Yuan) }\end{array}$} & \multicolumn{3}{|c|}{$\begin{array}{l}\text { Primary medical institutions } \\
\text { (Billions of Yuan) }\end{array}$} \\
\hline & $\begin{array}{l}\text { Actual } \\
\text { value }\end{array}$ & $\begin{array}{l}\text { Simulation } \\
\text { value }\end{array}$ & $\begin{array}{c}\text { Error } \\
(\%)\end{array}$ & $\begin{array}{c}\text { Actual } \\
\text { value }\end{array}$ & $\begin{array}{l}\text { Simulation } \\
\text { value }\end{array}$ & $\begin{array}{c}\text { Error } \\
(\%)\end{array}$ \\
\hline 2013 & 1693.65 & 1731.22 & 2.22 & 338.36 & 337.72 & -0.19 \\
\hline 2014 & 1952.42 & 1962.74 & 0.53 & 364.17 & 378.87 & 4.04 \\
\hline
\end{tabular}

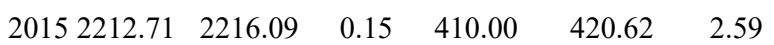

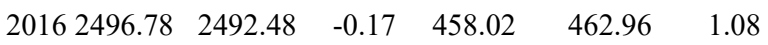

$\begin{array}{llllll}20172790.13 & 2793.11 & 0.11 & 521.84 & 505.91 & -3.05\end{array}$

As shown in Table 1, except for $2.2 \%$ in 2013 , the cost error of higher-level medical institutions in other years is less than 1\%; although the cost error of primary medical institutions in 2014 is $4.04 \%$, the overall error is within the acceptable range. Using the cost of different levels of medical system in 2013-2017 to verify the authenticity of the model, the overall error rate of the model is small, which indicates that the simulation model we have established is practical.

\section{Simulation analysis}

Graded diagnosis and treatment will increase the proportion of visits to primary care institutions, affect the cost of primary care institutions and higher-level medical institutions, and ultimately affect the total cost of medical institutions. This study assumes that the proportion of primary care visits per year will increase by $5 \%, 10 \%$, and $15 \%$ from 2017 to 2021 . Based on this, the cost and total cost of the medical institutions at different levels in the five-year increase ratio are calculated. At the same time, these values are compared with the original values (real and simulated values) to assess the impact of the increase in the number of primary consultations on total cost optimization.

First, calculate the cost of the primary medical institutions in different years and make a fold line, as shown in Figure 6.

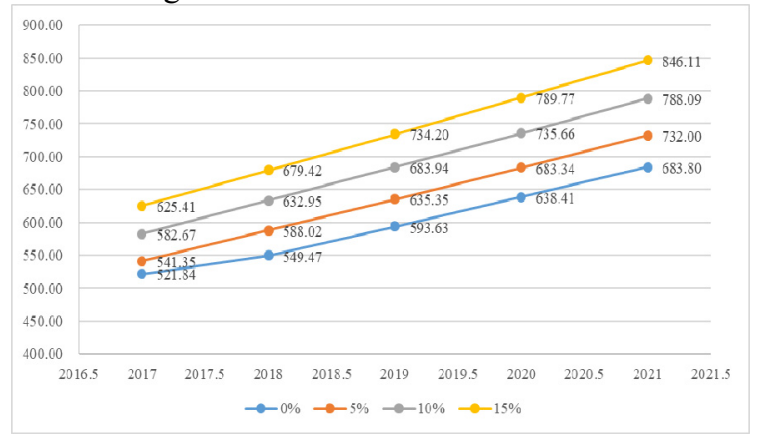

Figure.6. Sensitivity analysis of the cost of primary care institutions (Billions of Yuan)

Figure 6 shows that the increase in the proportion of people attending the primary level will lead to an increase in the cost of primary care. Moreover, the higher the proportion of people attending the primary level, the higher the cost of primary care. According to calculations, when the proportion increases by $5 \%$, the cost of primary medical institutions will increase by 
$3.74 \%, 7.02 \%, 7.03 \%, 7.04 \%$, and $7.05 \%$ from 2017 to 2021 respectively. When the proportion increases by $10 \%$, the cost of medical institutions increased by $11.66 \%, 15.19 \%, 15.21 \%, 15.23 \%$, and $15.25 \%$, respectively. When the proportion increased by $15 \%$, the cost increased by $19.85 \%, 23.65 \%, 23.68 \%$, $23.71 \%$, and $23.74 \%$, respectively. It can be seen that, except for 2017, the increase in the cost of other years is relatively stable.

Calculate the cost of the higher-level medical institutions in different years and make a fold line, as shown in Figure 7.

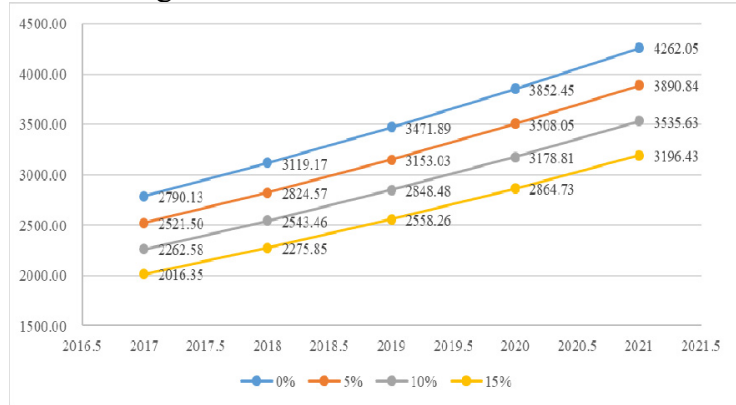

Figure.7. Sensitivity analysis of the cost of superior medical institutions (Billions of Yuan)

Figure 7 shows that the increase in the proportion of patients attending the primary level will lead to a decline in the cost of higher-level medical institutions. Moreover, the higher the proportion of the number of people attending the primary level, the lower the cost of the higher-level medical institutions. According to the calculation, when the proportion of the number of people attending the primary level increased by $5 \%$, the cost of the higher-level medical institutions decreased by $9.63 \%, 9.44 \%, 9.18 \%, 8.94 \%$, and $8.71 \%$ from 2017 to 2021; when the proportion increased by $10 \%$, In the past five years, the cost of higher-level medical institutions decreased by $23.32 \%, 18.46 \%$, $17.96 \%, 17.49 \%$, and $17.04 \%$, respectively. When the proportion increased by $15 \%$, they decreased by $27.73 \%, 27.04 \%, 26.32 \%, 25.64 \%$, and $25.00 \%$, respectively. It can be seen that when the proportion of the number of visits to the primary level is increased, the proportion of the cost of the higher-level medical institutions is also relatively stable.

Calculate the total cost of the higher-level medical institutions in different years and make a polyline, as shown in Figure 8.

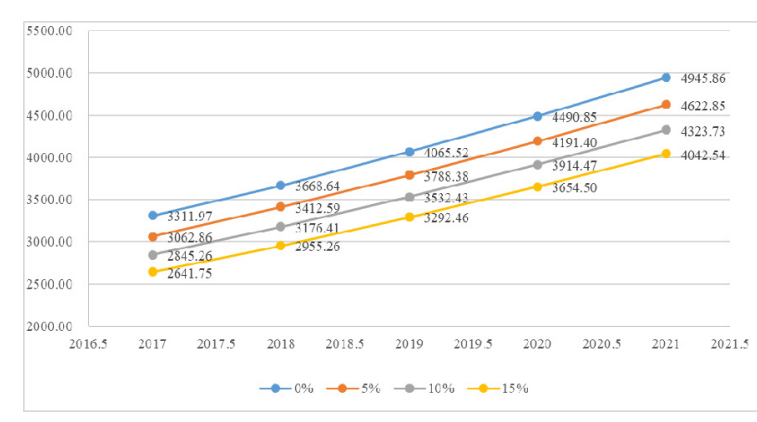

Figure.8. Sensitivity analysis of total cost of medical institutions (Billions of Yuan)

At the same time, calculate the amount and percentage of total cost reductions of medical institutions in different situations, as shown in Table 2 .

Table.2. The amount and percentage of total cost reduction of medical institutions in different situations

\begin{tabular}{ccrrr}
\hline & & $5 \%$ & $10 \%$ & $15 \%$ \\
\hline \multirow{2}{*}{2017} & Amount(Billions of Yuan) & 249.11 & 466.71 & 670.22 \\
& Rate (\%) & 7.52 & 14.09 & 20.24 \\
\multirow{2}{*}{2018} & Amount(Billions of Yuan) & 256.05 & 492.23 & 713.38 \\
& Rate (\%) & 6.98 & 13.42 & 19.45 \\
2019 & Amount(Billions of Yuan) & 277.14 & 533.09 & 773.06 \\
\multirow{2}{*}{2020} & Amount(Billions of Yuan) & 299.45 & 576.38 & 836.35 \\
& Rate (\%) & 6.67 & 12.83 & 18.62 \\
2021 & Amount(Billions of Yuan) & 323.01 & 622.13 & 903.32 \\
& Rate (\%) & 6.53 & 12.58 & 18.26 \\
\hline
\end{tabular}

Figure 8 shows that when the proportion of primary consultations increased by $5 \%, 10 \%$, and $15 \%$, the total cost of medical institutions was lower than the original value, and the higher the proportion, the lower the total cost. It can be seen from the table 2 that when the proportion of the number of visits to the primary level is increased by $5 \%$, the total cost of medical institutions in 2017-2021 is about 7\% lower than the original value, and the average annual reduction is 280 billion yuan. When the proportion increases by $10 \%$, the total cost of medical institutions in 2017-2021 is about $13 \%$ lower than the original value, with an average annual reduction of 540 billion yuan. When the proportion increases by $15 \%$, the total cost of medical institutions in $2017-2021$ is about $19 \%$ lower than the original value, with an average annual reduction of 780 billion yuan. In 2021, the decline was the biggest, reaching 903.32 billion yuan. 
Through sensitivity analysis, the cost of primary care institutions in 2017-2021 will increase due to the increase in the number of people attending the primary level in 2017-2021, and the proportion of cost increase is relatively stable. Similarly, an increase in the number of visits to the primary level will result in a steady decline in the cost of higher medical institutions during the period 2017-2021. Moreover, due to the large gap between the cost per capita of the higher hospital and the primary medical institution, the cost of the medical institution is much lower than that of the primary medical institution, which leads to a decline in the total cost of medical care. And the higher the proportion of the number of visits to the primary level, the more the total cost of medical care is saved. If the trend of increasing the number of people attending the primary level remains unchanged over time, it will be able to bring about a significant reduction in the total cost of Chinese medical institutions. If the annual 15\% increase is maintained until 2021, the total cost savings for Chinese medical institutions will reach 903.32 billion yuan.

\section{Conclusion}

The purpose of graded diagnosis and treatment is to increase the rate of primary consultation at the grassroots level, and the increase in the proportion of people attending the primary level can reduce the total cost of medical institutions. On this basis, this study constructs a system dynamics model of the relationship between the number of visits to medical institutions at all levels and its cost, and verifies the validity of the model by comparing the actual data with the simulated data from 2013 to 2017.

Secondly, the sensitivity analysis of the ratio of cost to grassroots visits in 2017-2021 is made. The results of the analysis show that although the cost of primary medical institutions will increase due to the increase in the number of visits, the cost of higher-level medical institutions will be significantly reduced, resulting in a reduction in the total cost of the entire medical system. Moreover, the higher the proportion of the number of people attending the primary level, the lower the total cost reduction. If the proportion of primary care in 2017 increases by $15 \%$, and this trend is maintained until 2021, the total cost saved by medical institutions in 2021 will be as high as 903.32 billion yuan.

\section{References}

[1] Sturmberg J P , Martin C M , Katerndahl D A . Systems and Complexity Thinking in the General Practice Literature: An Integrative, Historical Narrative Review[J]. The Annals of Family Medicine, 2014, 12(1):66-74.
[2] Yu W, Li M , Ge Y, et al. Transformation of potential medical demand in China: A system dynamics simulation model[J]. Journal of Biomedical Informatics, 2015, 57.

[3] Márta Somogyvári. The Costs of Organisational Injustice in the Hungarian Health Care System[J]. Journal of Business Ethics, 2013, 118(3):543-560.

[4] Ahmad, S. The Cost-Effectiveness of Raising the Legal Smoking Age in California[J]. Medical Decision Making, 2005, 25(3):330-340.

[5] Edelstein B L , Hirsch G, Frosh M, et al. Reducing early childhood caries in a Medicaid population[J]. The Journal of the American Dental Association, 2015, 146(4):224-232.

[6] Wakeland W, Nielsen A, Schmidt T D , et al. Modeling the Impact of Simulated Educational Interventions on the Use and Abuse of Pharmaceutical Opioids in the United States: A Report on Initial Efforts[J]. Health Education \& Behavior, 2013, 40(1 Suppl):74S-86S.

[7] Ishikawa T, Fujiwara K, Ohba H , et al. Forecasting the regional distribution and sufficiency of physicians in Japan with a coupled system dynamics-geographic information system model[J]. Human Resources for Health, 2017, 15(1):64.

[8] Ishikawa T , Ohba H, Yokooka Y , et al. Forecasting the absolute and relative shortage of physicians in Japan using a system dynamics model approach[J]. Human Resources for Health, 2013, 11(1):41.

[9] Barber P , Beatriz González López-Valcárcel. Forecasting the need for medical specialists in Spain: application of a system dynamics model[J]. Human Resources for Health, 2010, 8(1):24.

[10] Vanderby S A , Carter M W , Latham T , et al. Modeling the Cardiac Surgery Workforce in Canada[J]. Annals of Thoracic Surgery, 2010, 90(2):467-473.

[11] Wang L C , Cheng C Y, Tseng Y T , et al. Demand-pull replenishment model for hospital inventory management: a dynamic buffer-adjustment approach[J]. International Journal of Production Research, 2015, 53(24): 14.

[12] Senese F , Tubertini P , Mazzocchetti A, et al. Forecasting future needs and optimal allocation of medical residency positions: The Emilia-Romagna Region case study[J]. Human Resources for Health, 2015.

[13] Diaz R, Behr J G, Tulpule M. A system dynamics model for simulating ambulatory health care demands $[\mathrm{J}]$. Simulation in Healthcare, 2012, 7(4): 243-250.

[14] Khanmohammadi S, Farahmand H, Kashani H. A system dynamics approach to the seismic resilience enhancement of hospitals[J]. International journal of disaster risk reduction, 2018, 31: 220-233.

[15] Wenya Y, Yipeng L , Chaoqun H, et al. Research of an emergency medical system for mass casualty incidents in Shanghai, China: a system dynamics model[J]. Patient Preference and Adherence, 2018, Volume 12:207-222. 